\section{$\mathbf{m} / \mathbf{s}$}

médecine/sciences $1997 ; 13: 763-5$

\title{
COMMUNICATIONS ET TRANSMISSION DES SIGNAUX VASCULAIRES
}

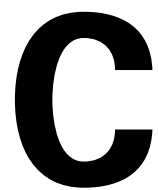

omme pour beaucoup d'autres disciplines, la physiologie, la physiopathologie, la pharmacologie... et la génétique du système vasculaire, ont bénéficié ces dernières années des nouvelles connaissances concernant les bases moléculaires de la transmission des signaux : communications entre cellules, communications entre cellules et matrices, principales voies intracellulaires de transmission du signal. Leurs applications au tissu vasculaire permettent d'aborder sous un jour nouveau les grandes questions que sont la régulation de la vasomotricité et les adaptations structurales des vaisseaux à de nouvelles conditions de fonctionnement. Cet approfondissement permet également de développer de nouvelles molécules et de nouveaux vecteurs, d'intérêt pharmacologique, voire thérapeutique. Comparé à d'autres systèmes, le système vasculaire n'en conserve pas moins certaines spécificités qui font l'intérêt particulier de son étude.

Les vaisseaux sont des organes tubulaires compartimentés juxtaposant au compartiment sang-endothélium, un compartiment médial constitué essentiellement de cellules musculaires lisses et de matrice extracellulaire, et un compartiment externe ou adventice constituant l'interface avec les tissus environnants. Le compartiment médial est le compartiment effecteur fonctionnel de la vasomotricité (contraction et relaxation de la cellule musculaire lisse), et celui dans lequel se produisent, en outre, les modifications de la trophicité vasculaire : hypertrophie et prolifération des cellules musculaires lisses, biosynthèse et destruction de la matrice... L'activité fibrosante de l'adventice participe également, dans certains cas, au modelage physiologique ou au remodelage pathologique des vaisseaux. Le compartiment sang-endothélium intervient dans les phénomènes d'angiogenèse. Le compartiment médial perçoit des signaux provenant de deux directions, soit centrifuges, allant du sang circulant vers la paroi, généralement relayés par l'endothélium quand celui-ci est intact, soit centripètes provenant de l'adventice et des tissus environnants. En cas de défaut fonctionnel ou d'absence structurale de l'endothélium, il se produit des phénomènes de décompartimentation centrifuge modifiant profondément la transmission des signaux véhiculés par le compartiment sanguin et la cellule musculaire lisse vasculaire, voire la matrice. De même, toute modification de fonction ou de structure d'un tissu retentit de façon centripète, directement ou indirectement, sur les vaisseaux qui l'irriguent.

Les voies moléculaires de transmission intracellulaire du signal réglant les fonctions vasculaires sont celles reconnues dans d'autres systèmes : voies des phospholipases, des nucléotides cycliques, des tyrosine kinases... Là également, ces signaux sont spécifiques du compartiment cellulaire vasculaire dans lequel ils sont engendrés. Par exemple, l'activation de la voie de la phospholipase $\mathrm{C}$ élève la 
concentration de calcium aussi bien dans la cellule musculaire lisse que dans la cellule endothéliale. Mais, dans la cellule musculaire lisse, ce signal aboutit directement à la contraction (couplage pharmacomécanique, voir l'article de G. Loirand et al. [1]), alors que, dans la cellule endothéliale, ce même signal augmente la production de monoxyde d'azote, et entraîne indirectement la vasodilatation. Finalement, une même molécule couplée à cette voie de transmission du signal peut être ambiguë en termes de réponse fonctionnelle pariétale (vasoconstriction contre vasodilatation), selon le compartiment dans lequel elle est majoritairement produite.

La majorité des signaux du couplage pharmaco-mécanique de contraction de la cellule musculaire lisse sont des signaux centripètes transmis de l'extérieur vers l'intérieur du vaisseau. Cela est principalement vrai pour le système nerveux sympathique et le système rénine/angiotensine, deux des principaux régulateurs du tonus contractile vasculaire. Les catécholamines libérées au niveau des varicosités terminales des axones sympathiques, présentes à l'interface adventice/média, sont responsables du tonus musculaire lisse d'origine sympathique. Il en est de même des autres neuromédiateurs vasomoteurs, y compris du monoxyde d'azote d'origine neuronale, vasodilatateur. De même, ce ne sont pas les angiotensines apportées par le plasma, mais plus probablement celles qui sont produites dans le tissu, à partir des protéines absorbées dans le tissu (rénine, angiotensinogène) qui sont responsables du tonus musculaire lisse dépendant de ce système.

Une question qui reste posée par le couplage pharmaco-mécanique est l'importance des phénomènes d'amplification des signaux dans la cellule musculaire lisse. En effet, on comprend mal comment aussi peu de ligands, à demi-vie si courte (angiotensine, noradrénaline, NO) sont capables d'engendrer des variations de fonction aussi importantes et aussi prolongées. C'est une des fonctions même de ces voies de transmission du signal que d'amplifier les signaux reçus, par le jeu d'activités enzymatiques capables d'agir en synergie sur plusieurs cibles. La mobilisation du calcium et la sensibilisation de l'appareil contractile, au cours du couplage pharmaco-mécanique, en sont un bon exemple (voir les articles de G. Loirand et al. et d'A. Fattoum [1, 2]). Une autre spécificité de la physiologie vasculaire dans ce domaine est le fonctionnement syncytial des cellules musculaires lisses. A partir d'une jonction neuro-musculaire, le signal de contraction diffuse à un ensemble de cellules musculaires lisses par l'intermédiaire de connexines (en particulier, la connexine 43 pour les cellules musculaires lisses $)\left(\mathrm{m} / \mathrm{s} n^{\circ} 5\right.$, vol. 1 , p. 706) [3], constituants des jonctions communicantes (gap junctions) qui laissent passer la plupart des seconds messagers intracellulaires.

Des modifications intrinsèques, génétiquement déterminées, même minimes, de ces signaux amplificateurs dans la cellule musculaire lisse vasculaire pourraient être le fondement du déterminisme génétique de l'hypertension artérielle essentielle, chez l'animal comme chez l'homme. Cependant, si nous connaissons bien les bases moléculaires de certaines anomalies de communication responsables d'hypertensions (hyperréninisme rénovasculaire, anomalies génétiquement déterminées de l'angiotensinogène, hyper-minéralocorticisme tumoral et génétique, phéochromocytome), les anomalies intrinsèques de la transmission du signal dans les cellules effectrices, suggérées par la physiopathologie, restent à définir à un niveau moléculaire. Chez le rat génétiquement hypertendu, les cellules musculaires lisses en culture primaire n'ont pas le même comportement prolifératif que les cellules provenant d'une souche normotendue, alors même que les conditions de l'environnement sont identiques.

Des résultats semblables ont été récemment rapportés chez l'homme [4]. Parmi les voies de signalisation vasculaires, il est apparu récemment $[5,6]$ que l'activation d'enzymes responsables de la production de radicaux libres (NADPH oxydase) pourrait faire le lien entre l'hypertension artérielle, les peptides vasopresseurs et le risque athéroscléreux.
La circulation pulmonaire est un bon exemple de communication centripète. Dans leur article, Serge Adnot et al. [7] montrent que la composition des gaz inspirés modifie de façon importante la fonction contractile puis la structure des artérioles pulmonaires. Ils introduisent le concept que l'hypoxie par elle-même peut induire des modifications fonctionnelles puis phénotypiques des cellules musculaires lisses, capables de remodeler les vaisseaux de façon pathologique. Le monoxyde d'azote, inspiré à des fins thérapeutiques, induit également des communications centripètes dans les artérioles pulmonaires, capables de s'opposer aux effets de l'hypoxie. Le rôle de l'hypoxie comme signal du remodelage vasculaire est repris par Christian Frelin et al. [8] qui montrent le rôle propre de ce signal dans l'induction physiologique, pathologique, voire thérapeutique de l'angiogenèse au travers de la régulation d'expression du VEGF (vascular endothelial growth factor).

Beaucoup de connaissances restent à acquérir concernant la transduction du signal secondaire à l'hypoxie dans le système vasculaire et de ses interactions avec les autres voies de transmission du signal. Comme le montrent ces deux articles, cette voie de transmission du signal joue probablement un rôle majeur dans de nombreuses et très diverses situations pathologiques (hypoxie respiratoire, ischémie musculaire, rétinopathie diabétique, varices, tumorigenèse) impliquant un remodelage vasculaire souvent intense contribuant à l'évolution pathologique, en réponse à des signaux centripètes provenant majoritairement du tissu environnant. Beaucoup reste encore à dévoiler sur les signaux et les communications propres à l'angiogenèse [9]. A l'opposé des facteurs angiogéniques, on a décrit récemment des facteurs anti-angiogéniques (thrombospondine, TGF-b, interleukine-12) dont certains sont des fragments peptidiques de protéines connues obtenus par clivage enzymatique : plasminogène $\rightarrow$ angiostatine, collagène $\rightarrow$ endostatine, fibronectine $\rightarrow 29 \mathrm{~K}$ fibronectine, prolactine $\rightarrow 16 \mathrm{~K}$ PRL) [10]. On ne connaît encore rien du mode 
d'action de ces molécules anti-angiogéniques ni de la transmission intracellulaire de leur signal dans le système vasculaire. Une des questions qui reste posée est pourquoi l'induction de facteurs de croissance angiogéniques par l'hypoxie est relativement inefficace à compenser complètement l'ischémie musculaire dans des situations pathologiques comme l'angor coronarien ou l'ischémie chronique des membres inférieurs.

Une des spécificités du tissu vasculaire, qu'il partage avec le tissu cardiaque, est de percevoir et d'intégrer en permanence les signaux de nature mécanique. Le muscle cardiaque est une pompe phasique génératrice de contraintes dans le système artériel. Ces contraintes sont de deux types, soit en rapport avec l'énergie potentielle (tension), soit en rapport avec l'énergie cinétique (cisaillement), engendrées par la contraction cardiaque.

Ces contraintes hémodynamiques, correspondant à des signaux centrifuges, induisent dans la paroi artérielle des réactions capables d'en modifier la fonction et la structure. La contrainte tensionnelle est essentiellement perçue par les cellules musculaires lisses de la média, la contrainte de cisaillement par les cellules endothéliales. Alain Tedgui et al. [11] montrent à quel point les bases moléculaires de la mécano-transduction se sont enrichies ces dernières années. Si ces contraintes peuvent jouer un rôle pathologique dans certaines situations, la mécano-transduction, en interaction avec la spécificité des communications entre cellules et matrice, joue un rôle physiologique essentiel dans la différenciation phénotypique des cellules vasculaires [12].

L'absence de contraintes mécaniques est probablement un des principaux déterminants de la dédifférenciation des cellules vasculaires lors de leur mise en culture. In vivo, les contraintes mécaniques sont vraisemblablement parmi les principaux déterminants du remodelage vasculaire dont les différentes formes et les différents déterminants sont de mieux en mieux décrits (voir l'article de Stéphane Laurent et al. [13]).

Les rôles protecteurs de l'imprégnation œstrogénique dans l'athérosclé- rose, et peut-être délétères dans la pathologie veineuse, sont abordés dans les articles de Jean-François Arnal et al. et de Martine PerrotApplanat [14, 15]. Là également, les bases moléculaires de l'action de ces hormones sur le tissu vasculaire sont de mieux en mieux connues. Mettre à jour les signaux cellulaires induits par les hormones a une importance considérable en thérapeutique, voire en santé publique (traitement substitutif postménopause).

L'interaction des hormones stéroïdes avec le potentiel oxydatif des cellules vasculaires est probablement une des clés pour comprendre leur rôle protecteur dans l'athérosclérose. L'effet paracrine de facteurs angiogéniques, sécrétés par les cellules hormonodépendantes du tractus de la reproduction sur le remodelage vasculaire au cours du cycle menstruel et de la grossesse reste très mal connu.

D'autres points importants de la physiopathologie vasculaire sont en pleine évolution scientifique. Les composantes moléculaires de la matrice extracellulaire vasculaire, leur biosynthèse, leur dégradation fonctionnelle (glycations) et structurale (protéases), leur rôle comme agents de communication avec les cellules vasculaires, comme participants de la pathologie athéromateuse (rupture de plaques, anévrismes), comme bases moléculaires d'anomalies génétiques (syndromes de Marfan, William et Beuren...), comme cibles thérapeutiques, font partie de cette évolution récente et représentent probablement de quoi alimenter un prochain numéro de médecine/sciences

\section{TIRÉS À PART}

J.B. Michel.

\section{RÉFÉRENCES}

1. Loirand G, Lompré AM, Savineau JP, Pacaud $\mathrm{P}$. Tonus des muscles lisses vasculaires : transmissions du signal dépendantes et indépendantes du $\mathrm{Ca}^{2+}$. Med Sci 1997 ; 13 : 766-76.

2. Fattoum A. Régulation de la contraction du muscle lisse. Med Sci 1997 ; 13 : 777-89.

3. Christ GJ, Spray DC, El-Sabban M, Moore LK, Brink PR. Gap junctions in vascular tissues. Evaluating te role of intercellular communication in the modulation of vasomotor tone. Circ Res $1996 ; 79: 631-46$.

4. Pietruck F, Moritz A, Montemurro M, Sell A, Busch S, Rosskopf D, Virchow S, Esche H, Brockmeyer N, Jakobs KH, Siffert W. Selectively enhanced cellular signaling by $\mathrm{Gi}$ proteins in essential hypertension. Circ Res 1996 ; 79 : 974-83.

5. Rajagopalan S, Kurz S, Münzel T, Tarpey M, Freeman BA, Griendling KK, Harrison DG. Angiotensin II mediated hypertension in the rat increases vascular superoxide production via menbrane NADH/NADPH oxidase activation : contribution to alterations of vasomotor tone. J Clin Invest 1996 ; 97 : 1916-23.

6. Fukui T, Ishizaka N, Rajagopalan S, Laursen JB, Capers Q, Taylor RW, Harrison DG, de Leon H, Wilcox JN, Griendling KK. p22phox mRNA expression and NADPH oxidase activity are increased in aortas from hypertensive rats. Circ Res 1997 ; 80 : 45-51.

7. Adnot S, Raffestin B, Dinh-Xuan AT. NO et hypertension artérielle pulmonaire. Med Sci 1997 ; 13 : 820-9.

8. Frelin C, Ladoux A, Bauters C. VEGF : médiateur de l'angiogenèse hypoxique. $\mathrm{Med} \mathrm{Sci}$ 1997 ; 13 : 886-91.

9. Schaper W, Ito WD. Molecular mechanism of coronary collateral vessel growth. Circ Res $1996 ; 79$ : $911-9$

10. Hanahan D, Folkman J. Patterns and emerging mechanisms of the angiogenic switch during tumorigenesis. Cell 1996; 86 : 353-64.

11. Tedgui A, Bardy N, Lévy B. Transmission des signaux mécaniques dans les cellules vasculaires. Med sci 1997 ; 13 : 790-8.

12. Owens GK. Role of mechanical strain in regulation of differentiation of vascular smooth muscle cells. Circ Res $1996 ; 79: 1054-5$.

13. Laurent S, Girerd X, Benetos A, DanielLamazière JM, Lacolley P. Physiopathologie du remodelage artériel dans l'hypertension artérielle. Med Sci 1997 ; 13 : 809-19.

14. Arnal JF, Elhage R, Faye JC, Bayard F. Quelle est la cible des œestrogènes dans la paroi artérielle ? Med Sci 1997 ; 13 : 881-5.

15. Perrot-Applanat M. Stéroïdes ovariens et système vasculaire : rôle dans la prolifération vasculaire et implication en pathologie veineuse. Med Sci 1997; 13 : 830-7. 